\title{
Quality Control in Endoscopy Unit: Safety Considerations for the Patient
}

\author{
Jean-Francois Rey \\ Institut Arnault Tzanck \\ Hepato-Gastroenterology Department \\ 06721 St Laurent du Var Cedex \\ France
}

\section{Introduction}

Safe endoscopic procedures have been a daily concern in every endoscopy unit since the early eighties. At first range, prevention of crossed contamination was the primary goal for endoscopy units' director. But, step after step, all parts of endoscopy procedures have to be monitored and the use of protocols is now mandatory. In this chapter, we will review: patient information and protocol of all endoscopy related procedures, check list in endoscopic theatre, control in endoscope maintenance and the importance of audit in endoscopy unit.

\section{Patient information}

In the last decade, patient information has been a very hot topic in case of complications issues. In some countries, it is the most important point in case of malpractice. The relationship between practitioners and patients has changed and the paternalist time is behind us. Patients are well informed, perhaps too much in some cases, through multiple media and mainly Internet.

Patient information is linked very closely with oral and written information. As proved by (Felley et al., 2008) in a randomized trial between combined written and oral information prior to a gastrointestinal endoscopy versus oral information alone. Written and oral is better than oral only. Written information should develop: preparation of the procedure, expectations and risks. It is important to rely on this issue on national endoscopy and gastroenterology scientific societies. The leaflets are written with accuracy and caution but is will be a mistake to handle it to the patient without oral comments, sometimes asking him to sign by a staff nurse. Oral information has to be adapted to the patient level of knowledge, language and culture; it is sometimes difficult. A letter to the patients' general practitioner is a wise mean to complete patient information and it is a proof in the practitioner file.

The content of information should deal with the following points:

- Pre-procedural considerations:

- Comorbidity, body habitus (mobility, airway)

- Anticoagulation and antiplatelet therapy

- $\quad$ Specific considerations for ERCP 
- Radiation with events relying to pregnancy

- Complications

- For colonoscopy, considerations have to be underlined on:

- Bowel preparation

- Cardiac and renal functions with issue related to the preparation in case of colonic lavage

- $\quad$ Post procedure informations are mandatory:

- Drowsiness: driving, operating machine, complete stases,

- Side effects and complications: chest pain, bleeding, vomiting, breathless, abdominal pain or fever. In case of any discomfort or complications, how the patient could contact the endoscopy unit or emergency department and bring the suitable endoscopy report.

- Follow-up appointment

- Recommencing if necessary, anticoagulation and antiplatelet therapy.

For more risky procedures as ERCP and therapeutic biliary pancreatic endoscopy, ASGE makes a detailed recommendation (Table 1). If variances occur outside of these recommendations, careful medical justification should be recorded.

A. Jaundice thought to be the result of biliary obstruction

B. Clinical and biochemical or imaging data suggestive of pancreatic or biliary tract disease

C. Signs or symptoms suggesting pancreatic malignancy when direct imaging results are equivocal or normal

D. Pancreatitis of unknown etiology

E. Preoperative evaluation of chronic pancreatitis or pancreatic pseudocyst

F. Sphincter of Oddi manometry

G. Endoscopic sphincterotomy

1. Choledocholithiasis

2. Papillary stenosis or sphincter of Oddi dysfunction causing disability

3. Facilitate biliary stent placement or balloon dilatation

4. Sump syndrome

5. Choledochocele

6. Ampullary carcinoma in poor surgical candidates

7. Access to pancreatic duct

O. Stent placement across benign or malignant strictures, fistulae, postoperative bile leak, or large common bile duct stones

P. Balloon dilatation of ductal strictures

Q. Nasobiliary drain placement

R. Pseudocyst drainage in appropriate cases

S. Tissue sampling from pancreatic or bile ducts

T. Pancreatic therapeutics

VARIANCE REQUIRES JUSTIFICATION

Table 1. ASGE recommendation (Indications for ERCP) 
Finally all informations could be related in a consent form which deals with multiple issues. (Bottrell et al., 2000) underlining the main questions:

- What are the local legal requirements? This topic is highly variable to one country to another.

- Ethical requirements

- Physicians-Patients relationships

All elements related to the procedure has to be recorded (Elfant et al.,1995):

- Nature of the procedure and its handling.

- Procedure benefits: treat bleeding, identify source of bleeding, pain, discomfort, diagnosis, remove polyps, inserting stents.

- Procedure related risks: bleeding, infection, perforation, cardiopulmonary, allergy.

- Alternatives with disadvantages versus advantages. Do we have an alternative treatment?

Ideally, this information and consent form request have to be carried on days before procedure. The patient should have time to read, understand and make its mind. (Song et al., 2010) has recently published an inquiry on the benefit of the informed consent (Table 2).

Did you think that the informed consent was a useful and necessary procedure?

What did you think was the main objective of the informed consent procedure?

. Improving a patients' understanding

. Evidence of understanding and mutual agreement

. Protection of the legal rights of the doctors

. Senseless formality

Table 2. Informed Consent (Song et al., 2010)

\section{Protocols}

Written protocols have to be implemented in each endoscopy unit. It is the last way to keep high standard in a daily routine whatever the procedure is carried out by a senior or a junior endoscopist (Naylor et al., 2003; Faigel et al., 2006). In most of the cases, written protocols are issued from national or international guidelines but adapted to the local situation. Protocol means program for the systematic monitoring and evaluation of the various aspects of a project, service or facility to ensure that standards of quality are being met. The program for quality assurance has been described by ( $\mathrm{O}^{\prime}$ Mahony et al., 2000) with the following topics:

1. Improve the overall quality of care

2. Limit inappropriate procedures

3. Limit morbidity and mortality

4. Improve training in endoscopy

5. Limit patient complaints and litigation

6. Contain costs

7. All of the above 
In building your protocol, you should identify quality, indicators, members of your team and how to build the process. This lead to raise multiple questions (O'Mahony et al., 2000):

1. How to set it up so that it works in everyday practice?

2. What information do we need to collect and how should we analyse it?

3. How much will it cost, who will pay for it?

4. How do we set quality standards?

5. How do we deal with underperforming doctors?

Some are obvious as how to use properly and set adequately an electrosurgical unit in relation with the device (Rey et al., 2010). For therapeutic endoscopy a closed monitoring of patient coagulation is also mandatory as old patients are often treated with anticoagulation drugs or even more dangerous some very effective antiplatelet drug such as Clopidogrel. Condition risk for thromboembolic has been described by a national society (ASGE, 2009), (Table 3) as the embolism risk after a brief interruption of anticoagulation (Veitch et al., 2008), (Table 4). But we have to accept various recommendations for the same topic if guidelines have been designed by cardiology or gastroenterology scientific societies. Antibio-prophylaxy protocol has to be designed in requirements of national recommendations and adapted with a better knowledge of patient condition. Level of competency for endoscopists practitioners is also variable from one country to another as reminded by (Faigel et al., 2009), (Table 5).

\begin{tabular}{|c|c|}
\hline Higher-risk condition Low-risk condition & Low-risk condition \\
\hline $\begin{array}{l}\text { - Atrial fibrillation associated with valvular } \\
\text { heart disease, prosthetic valves, active } \\
\text { congestive heart failure, left ventricular } \\
\text { ejection fraction }<35 \% \text {, a history of a } \\
\text { thromboembolic event, hypertension, } \\
\text { diabetes mellitus, or age }>75 \mathrm{y}\end{array}$ & $\begin{array}{l}\text { - Uncomplicated or paroxysmal } \\
\text { nonvalvular atrial fibrillation }\end{array}$ \\
\hline - Mechanical valve in the mitral position & - Bioprosthetic valve \\
\hline $\begin{array}{l}\text { - Mechanical valve in any position and } \\
\text { previous thromboembolic event }\end{array}$ & - Mechanical valve in the aortic position \\
\hline - Recently ( $<1$ y) placed coronary stent & \\
\hline - Acute coronary syndrome & - Deep vein thrombosis \\
\hline $\begin{array}{l}\text { - Non stented percutaneous coronary } \\
\text { intervention after myocardial infarction }\end{array}$ & \\
\hline
\end{tabular}

Table 3. Condition risk for thromboembolic event (ASGE Standards of Practice Committee, 2009) 


\begin{tabular}{|c|c|}
\hline High risk & Low risk \\
\hline - VTE, embolic stroke $\leq 12 \mathrm{wk}$ ago & - DVT/PE $\geq 12$ wk ago \\
\hline - AF with valvular HD & - NV AF, no CCF/embolism \\
\hline $\begin{array}{l}\text { - Mechanical mitral/multiple valves, } \\
\text { previous TEE }\end{array}$ & - Bio-prosthetic valve \\
\hline - Thrombophilia & - Mechanical aortic valve \\
\hline \multirow[t]{3}{*}{$\begin{array}{l}\text { - IHD with bare stent } \leq 12 \text { wk ago } \\
\text {. DE stent } 6-12 \text { mo ago }\end{array}$} & - IHD, no coronary stents \\
\hline & - Cerebrovascular disease (CVD) \\
\hline & - PVD \\
\hline \multicolumn{2}{|c|}{$\begin{array}{l}\text { - Embolic risk if anticoagulation interrupted in low risk condition 1-2/1000 } \\
\text { - Avoid elective procedure for } 12 \mathrm{wks} \text { after acute DVT/PE }\end{array}$} \\
\hline
\end{tabular}

Table 4. Embolism risk after brief interruption of anticoagulation (Veitch et al., 2008)

\begin{tabular}{|lcccccc|}
\hline & USA * & Australia $=$ & Canada & Poland & India & Europe $\ddagger$ \\
Procedure & & & & & & \\
Sigmoidoscopy & 30 & & 30 & & & 50 \\
Colonoscopy & 140 & 100 to cecum & 150 & 500 & 120 & 150 \\
EGD & 130 & 200 & 150 & 500 & 190 & 200 \\
ERCP & 200 & 200 & 200 & 200 & 140 & 150 \\
EUS & 150 & 200 & & & & 150 \\
\hline
\end{tabular}

EGD, esophagogastroduodenoscopy; ERCP, endoscopic retrograde cholangiopancreatography; EUS, endoscopic ultrasonography

* American Society for Gastrointestinal Endoscopy (ASGE) Guideline

$=$ Colonoscopy: cecal intubation in $>90 \%$ of the last 50 logged procedures. ERCP: unassisted, with intact papilla, to include 80 sphincterotomies and 60 stent placements.

‡ European Board of Gastroenterology: colonoscopy numbers include polypectomy, and assume competency in EGD first (10).

Table 5. Threshold numbers of endoscopic procedures before competency can be assessed by direct observation or other objective measures, as required in different countries or regions. (Faigel et al., 2009).

\section{Time out}

Endoscopy procedures are very close to surgical procedures, it is why endoscopy suites have to be carried on as a surgical theatre taking in account endoscopy specificities (Table 6). Recently a new process has been issued as time out or also called "checklist" (Haynes et al., 2009). This process is issue from airplane pilot cockpit where, before each flight commandant and first officer revised orally and click on a sheet, all airplane equipment. Of course, it is much more easily with machine elements than with a human body. The main 
goal is to avoid errors in patient labelling, type of the procedures, where procedures should be applied, patient treatment and more specifically, anticoagulation and antiplatelet therapy. In endoscopy, the clinical benefit of the time out or checklist has to be proved but it becomes more important in a very busy unit.

\section{Sign in}

Before induction of anesthesia, members of the team (at least the nurse and an anesthesia professional) orally confirm that:

The patient has verified his or her identity, the surgical site and procedure, and consent.

The surgical site is marked or site marking is not applicable

The pulse oximeter is on the patient and functioning

All members of the team are aware of wheter the patient has a known allergy

The patient's airway and risk of aspiration have been evaluated and appropriate equipment and assistance are available

If there is a risk of blood loss of at least $500 \mathrm{ml}$ (or $7 \mathrm{ml} / \mathrm{kg}$ of body weight, in children), appropriate access and fluids are available

\section{Time out}

Before skin incision, the entire team (nurses, surgeons, anesthesia professionals, and any others participating in the care of the patient) orally:

Confirms that all team members have been introduced by name and role

Confirms the patient's identity, surgical site, and procedure

Reviews the anticipated critical events

Surgeon reviews critical and unexpected steps, operative duration, and anticipated blood loss

Anesthesia staff reviews concerns specific to the patient

Nursing staff reviews confirmation of sterility, equipment availability, and other concerns

Confirms that prophylactic antibiotics have been administrated $\leq 60 \mathrm{~min}$ before incision is made or that antibiotics are not indicated

Confirms that all essential imaging results for the correct patient are displayed in the operating room

\section{Sign out}

Before the patient leaves the operating room:

Nurse reviews items aloud with the team

Name of the procedure as recorded

That the needle, sponge, and instrument counts are complete (or not applicable)

That the specimen (if any), is correctly labelled, including with the patient's name

Wether there are any issues with equipment to be addressed

The surgeon, nurse and anesthesia professional review aloud the key concerns for the recovery and care of the patient

* the checklist is based on the first edition of the WHO Guidelines for Safe Surgery (Geneva, World Health Organization, 2008).

Table 6. Elements of the Surgical Safety Checklist* (Haynes et al., 2009) 


\section{Control in endoscope maintenance}

Quality control in servicing and repairing endoscopes is an important business for each endoscopy unit. It is why we have been required to write a guideline on behalf of ESGE (Rey et al., 2004). This work has been carried on with the help of the personal from three major endoscopes makers, Fujinon, Olympus and Pentax. But it is clear that repairing services could be provided both by endoscopes manufacturers and so called third party as independent repaired company.

In issuing guidelines on repairing endoscopes, the aim of the European Society of Gastrointestinal Endoscopy (ESGE) Guidelines Committee is to draw the attention of those who manage endoscopy units to the quality control requirements involved in having endoscopes repaired. Information on endoscope repair is important not only for medical doctors, nurses and assistants, but also for medical engineers and - even more so - for hospital administrators. The aim of these ESGE guidelines is to draw the attention of endoscope users to both the technical and the legal implications relating to endoscope repair. The sources of spare parts and the exact repair procedures used should be clearly stated in the repair contract. If second-hand spare parts are used, traceability and hygiene issues should be taken into consideration. For the ESGE, it is clear that repair services can be provided both by endoscope manufacturers and by other repair service providers (known as "third parties"), as long as they follow the same quality control processes and guarantees as those followed in endoscopy units.

The intention in these guidelines is to provide users of medical endoscopes with information about the appropriate selection of service providers, including information on how to reduce user liability relative to compliance with the Medical Device Directive (MDD) during the lifespan of endoscopic equipment and devices.

The Medical Device Directive (MDD) regulates the requirements for the design, manufacture and sale of new medical devices and equipment very precisely. A clear sign for the user that the equipment or device complies with MDD regulations is the CE mark. A reference to the original manufacturer is also provided by the identification label on each product. The basic aim of these measures is to ensure the safety of users, patients, and others, as well as to ensure compliance with technological standards. Before the first use of any new endoscope, the MDD requires proof that these requirements have been met, based on both clinical and nonclinical testing procedures. The original manufacturer maintains a master configuration file, which documents the design configuration, manufacturing configuration, and test results. This ensures seamless configuration control.

After the product has been sold, the liability and responsibility for ensuring safe usage of the product is transferred to the user. The degree of user liability is likely to depend on the contract between the user's organization and the selected service provider. The transfer of liability, and all associated risk, depends on the person or organization undertaking the service and on the way in which maintenance and repair work is carried out. If a failure in use occurs after maintenance or repair and this failure leads to serious injury to a patient or user, there is a greater likelihood that the user and service provider may be held legally responsible for any injuries caused, if the endoscope was not repaired in accordance with the manufacturer's instructions and operating standards.

Users need to ensure that they follow an appropriate strategy for maintaining and repairing the equipment. Options for maintenance and repair services include choosing either:

- The original manufacturer of the endoscope 
- An in-house biomedical engineering department

- An independent service provider

- A managed service provider.

Usually, the original manufacturer is the primary source for maintenance and repair services. However, not all manufacturers carry out repair and maintenance of their devices. Whoever is carrying out the maintenance and repair activity, the written service documentation provided by the manufacturer should always be used. If any organization other than the original manufacturer undertakes repair or maintenance work, the organization concerned should ensure that the work and inspection is carried out in accordance with the latest manufacturer's instructions and specifications, and according to defined standards.

The most important issues to be considered as selection criteria for any service provider are in the four core areas:

- General competence

- Workmanship

- Parts and materials

- Verification, inspection, and risk management.

However, in addition, the following criteria are important elements that should also be taken into account when choosing a service provider:

- A clear analysis and understanding of the relationship between apparent financial savings and increased liability risks, and calculation of total ownership costs relative to repair frequency

- $\quad$ The frequency with which maintenance and repairs are likely to be needed and the time required to respond to endoscope malfunction:

- Uptime requirements

- Requirements for loan equipment

- $\quad$ Requirements for a mobile service.

It is advisable for the owner of the endoscopic equipment to always make a clear contractual agreement with the chosen service provider. This agreement should clearly describe the level of service to be provided to the owner organization, and it is recommended that it should include:

- $\quad$ Reference to the original manufacturer's written instructions

- Information on availability, source, and traceability of spare parts

- Notification of any changes in parts, procedures, tests performed, etc., including the use of parts and methods other than those specified by and originating from the original manufacturer

- Details of the training and qualifications of the technicians and inspectors

- Details on the way in which requirements for adequate record-keeping regarding traceability are ensured

- Regulations on the use of subcontractors employed by the ser- vice provider.

A repair organization that complies with both the comprehensive legal and quality aspects as well as with specific customer requirements will be able to respond positively to the following questions.

\section{a) Competence}

- What is the experience and customer reputation that the ser- vice provider has in repairing and maintaining the specific type of endoscopic or electronic equipment? (List of customers) 
- Is a quality assurance system in place? (ISO 9001 - 2000 as a minimum; preferably ISO EN13488 to show compliance with MDD)

- How are the requirements for adequate record keeping and traceability ensured?

- Is the availability of loan equipment adequate to meet customer demand, and is the loan equipment chargeable or not?

- What is the response time and what are the regular service hours?

- Is a mobile service offered, and where is it located?

- Is the service organization sufficiently flexible to adapt to customers' uptime requirements?

- Is a risk management strategy in place?

- Does the service provider have reliable access to the original manufacturer's information (manuals, safety information, and device specifications) and spare parts?

b) Workmanship

- Are the original manufacturer's repair procedures in place and regularly updated, (preferably by the original manufacturer) and what is the source for the relevant documentation?

- How does the update procedure work, and how is it documented?

- Is a written agreement with the original manufacturer available?

- Is the level of documentation appropriate to the level of repair?

- Are the technicians regularly trained on the basis of the original manufacturer's manufacturing and service standards (preferably by the original manufacturer's approved trainers)?

- What certificates and records are available for repair and inspection training with regard to the specific equipment type and the extent of repair training that has been performed?

- What are the training intervals for technicians?

- What regular reviewing of the training level is done, and is it done at appropriate intervals?

- Are the tools that are being used in accordance with the original manufacturer's jigs and special tools specifications, or are they originally provided by the manufacturer?

- Are specific repair and inspection procedures in place, regularly updated, and followed (preferably by the origin manufacturer)?

- If repair and inspection procedures other than the original manufacturer's are being used:

- Are the methods and procedures documented and regularly updated and appropriately detailed on the basis of the required level of maintenance and repair?

- Is a system in place to inform the user organization of any alternative methods used?

- What certificates and records are available for inspection?

- Have the risks been identified and documented?

- Is a risk management strategy for this documentation in place?

c) Parts and Materials

- $\quad$ Are the criteria for the definition of part defects documented and verified as being in accordance with the original manufacturer's criteria and with MDD requirements?

- Are all the parts and materials used for repairs the certified original manufacturer's parts, and are they purchased from the original manufacturer or any authorized agency? 
- If no original parts and materials (e.g., adhesives) are used, is the service provider willing to certify the origin of the parts and materials used for repairs?

- Is a risk management strategy for these parts and materials in place and the appropriate documentation available?

- Have the risks been identified and documented?

- Are certificates of biocompatibility available?

- Do the parts and materials comply with the original manufacturer's specifications of compatibility with reprocessing agents?

- Is the sourcing of these parts covered by a contract (to ensure continuity of supply?

d) Verification, Inspection and Risk Management

- Have criteria for functionality and safety testing been defined, and are they available and verified as being in accordance with MDD requirements and the original manufacturing standards?

- Have the methods and equipment used for quality assurance inspection been defined and are they appropriate for verifying the quality of the repaired endoscope according to the origin manufacturer's standards? Is a quality assurance tracking report available?

- Will the repair contractor provide a statement of conformity with the original manufacturer's functional, safety, and quality standards after an endoscope has been repaired?

- Is the repair process documented and traceable?

- Process steps

- Technicians involved

- Parts used

- How long will the necessary documentation be kept available?

- What notification and approval procedures are in place to ensure that the user organization is aware of any changes in spare parts and methods other than those covered by the contract?

- With regard to the whole repair process, is a risk management system in place and who is the person responsible for it?

- Is a procedure in place for reporting conditions to the user organization that have a potential to cause a device failure or otherwise compromise the intended use of the endoscope?

\section{e) Technical consequences of any repairs services}

To carry out any repairs, it is necessary to disassemble an endoscope either partly or wholly. During this procedure, the functionality and condition of subcomponents or parts inside the endoscope can be inspected in addition to verifying the originally detected problem. Reassembling the parts, including internal adjustments of subcomponents, also requires specialized understanding and expertise in the manufacturer's procedures. Performing the repairs in accordance with the manufacturer's standards ensures the continuing functionality and safety of the endoscope.

If any changes are made during a repair procedure with regard to the materials, spare parts used, or characteristics of the medical endoscope, this may invalidate the related CE marking. In case of doubt, a new CE marking procedure carried out by a registered medical device manufacturer for the endoscope concerned will be required. 
Responsibility for the safety of the device lies primarily with the owner or his or her delegated person, regardless of who carries out any repairs.

Effectively, this means that in the event of endoscopic equipment being subjected to any work, processing, etc., that is not in accordance with the original manufacturer's specifications and/or quality standards, the equipment may have performance standards that deviate from (i.e., are inferior to) those for which the original CE approval by a notified body and its registration was issued.

This would therefore completely invalidate any continuing liability on the part of the original manufacturer even for equipment that might still normally be within the manufacturer's warranty period. Any liability for claims arising from subsequent malfunctioning or harm to patients and/or users would therefore pass to the owners of the equipment.

In summary, endoscopy maintenance is an important point in order to keep high standard endoscopy unit procedure. The organisation of repairing service may vary from one country to another depending of the local organisation in national legal requirement. For accessories, it is even more important with legal and technical concerns. With single use equipment, the safety is linked to one time use accessory, for reusable device, we should follow standard for cleaning and disinfection (Beilenhoff et al., 2008). Of course in many countries, single use accessories are reused, it becomes a legal issue if forbidden by law or a civil concern in case of inadequate reprocessing.

\section{Endoscopy unit audit}

This point has been particularly developed for monitoring of cleaning and disinfection procedures, but also, more generally in the British National Health Service (NHS) for all endoscopy units accredited for colorectal cancer screening (the JAG).

Cleaning and disinfection of endoscopes is a complex and multi-tasks process. Mistake in handling could occur at any steps. Manual pre-cleaning has to be developed on immediately after the end of the endoscopy procedure. Cleaning and disinfection could be achieved with manual cleaning or water disinfection machine. But in all cases, defects may occurred and lead to an infective process. For example, water supply or washer-disinfector could be infected with pseudomonas. It is why regular monitoring, every 3 months with specimen analysis at each step, is recommended. In case of reprocessing accessories, cleaning and disinfection have to be monitored very closely and it is why, in many endoscopy units, single use devices are a primary choice.

Due to the poor reputation of NHS, huge financial investments have been implemented five years ago in order to improve efficacy, safety and reduce waiting lists especially with the development of colorectal cancer screening policy. Each endoscopy unit had to develop accreditation process for all procedures, physicians have been also audited by senior endoscopists. In a couple of years, marked improvements have been obvious. This policy should be strongly recommended for each country (Faigel et al., 2009). Avoid side effects should be a daily goal for every endoscopy unit director but also for all practitioners and staff.

\section{Conclusion}

Quality control is becoming a very important point in each endoscopy unit. It is a multifactor process where medical and non-medical staffs have to work altogether very 
closely. It is also a primary goal and question issue for our patients and patients' associations: Doctor, is it safe to be scoped in your unit?

\section{References}

ASGE Standards of Practice Committee (2009). Management of antithrombotic agents for endoscopic procedures. Gastrointest Endosc. 70, 6, pp.1060-1070

Beilenhoff, U. et al. (2008). ESGE-ESGENA guideline: Cleaning and disinfection in gastrointestinal endoscopy-Update 2008. Endoscopy, 40, pp. 939-957

Bottrell, M. et al. (2000). Hospital informed consent for procedure forms. Arch Surg, 135, 1, pp. 26-33

Elfant, A. et al. (1995). Recall of informed consent after endoscopic procedures. Dis Col Rect, 38,1, pp. $1-3$

Faigel, D.O. et al. (2006). Quality indicators for gastrointestinal endoscopic procedures: an introduction. Gastrointest. Endosc. 63, (4 Suppl), pp. S3-9

Faigel D.O. et al. (2009). London OMED Guidelines for credentialing and quality assurance in digestive endoscopy. Endoscopy, 41, pp. 1069-1074

Felley, C. et al. (2008). Combined written and oral information prior to GI endoscopy compared with oral information alone: a randomized trial. BMC Gastroenterology, 8, pp. 22

Haynes A.B. et al. (2009). A surgical safety checklist to reduce morbidity and mortality in a global population. N. Engl J Med, 360, 5, pp.491-499

Joint Advisory Group on Gastrointestinal Endoscopy (the JAG). http:/ / www.thejag.org.uk

Naylor, G. et al. (2003). Setting up a quality assurance program in endoscopy. Endoscopy, 35, 8, pp.701-707

O'Mahony, S.; Naylor, G. \& Axon A. (2000). Quality assurance in gastrointestinal endoscopy. Endoscopy, 32, 6, pp. 483-488

Rey, J.F. et al. (2004). ESGE Guidelines for Quality Control in Servicing and Repairing Endoscopes. Endoscopy, 36, pp. 921-923

Rey, J.F. et al. (2010). European Society of Gastrointestinal Endoscopy (ESGE) guideline: the use of electrosurgical units. Endoscopy, 42, pp. 764-771

Song, J.H. et al. (2010). Acceptance and understanding of the informed consent procedure prior to gastrointestinal endoscopy by patients: a single-center experience in Korea. Korean J Intern Med, 25, 1, pp. 36-43

Veitch, A.M. et al. (2008). Guidelines for the management of anticoagulant and antiplatelet therapy in patients undergoing endoscopic procedures. Gut, 57, pp.1322-1329 


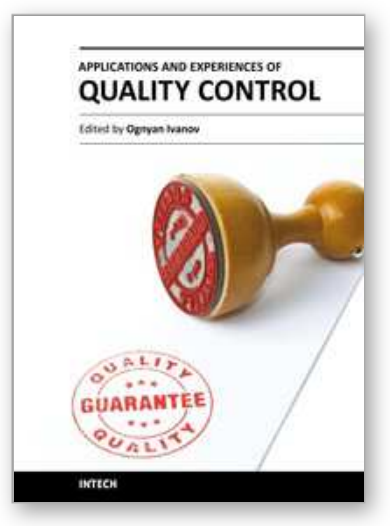

\section{Applications and Experiences of Quality Control \\ Edited by Prof. Ognyan Ivanov}

ISBN 978-953-307-236-4

Hard cover, 704 pages

Publisher InTech

Published online 26, April, 2011

Published in print edition April, 2011

The rich palette of topics set out in this book provides a sufficiently broad overview of the developments in the field of quality control. By providing detailed information on various aspects of quality control, this book can serve as a basis for starting interdisciplinary cooperation, which has increasingly become an integral part of scientific and applied research.

\section{How to reference}

In order to correctly reference this scholarly work, feel free to copy and paste the following:

Jean-Francois Rey (2011). Quality Control in Endoscopy Unit: Safety Considerations for the Patient, Applications and Experiences of Quality Control, Prof. Ognyan Ivanov (Ed.), ISBN: 978-953-307-236-4, InTech, Available from: http://www.intechopen.com/books/applications-and-experiences-of-qualitycontrol/quality-control-in-endoscopy-unit-safety-considerations-for-the-patient

\section{INTECH}

open science | open minds

\author{
InTech Europe \\ University Campus STeP Ri \\ Slavka Krautzeka 83/A \\ 51000 Rijeka, Croatia \\ Phone: +385 (51) 770447 \\ Fax: +385 (51) 686166 \\ www.intechopen.com
}

\author{
InTech China \\ Unit 405, Office Block, Hotel Equatorial Shanghai \\ No.65, Yan An Road (West), Shanghai, 200040, China \\ 中国上海市延安西路65号上海国际贵都大饭店办公楼405单元 \\ Phone: +86-21-62489820 \\ Fax: +86-21-62489821
}


(C) 2011 The Author(s). Licensee IntechOpen. This chapter is distributed under the terms of the Creative Commons Attribution-NonCommercialShareAlike-3.0 License, which permits use, distribution and reproduction for non-commercial purposes, provided the original is properly cited and derivative works building on this content are distributed under the same license. 